\title{
Correction to "Commentary on: Does COVID19 Infect the Brain? If So, Smokers Might Be at a Higher Risk"
}

In the above article [Speth RC (2020) Mol Pharmacol, 98: 382-383; DOI: https://doi.org/10. 1124/molpharm.120.000049], the older definition of prolylcarboxypeptidase (PrCP) "angiotensinase A" was accidentally listed in Fig. 1. It should be "angiotensinase C." The corrected Fig. 1 is provided below. The HTML and PDF versions of the article have been corrected.

The authors apologize for any inconvenience this may have caused.

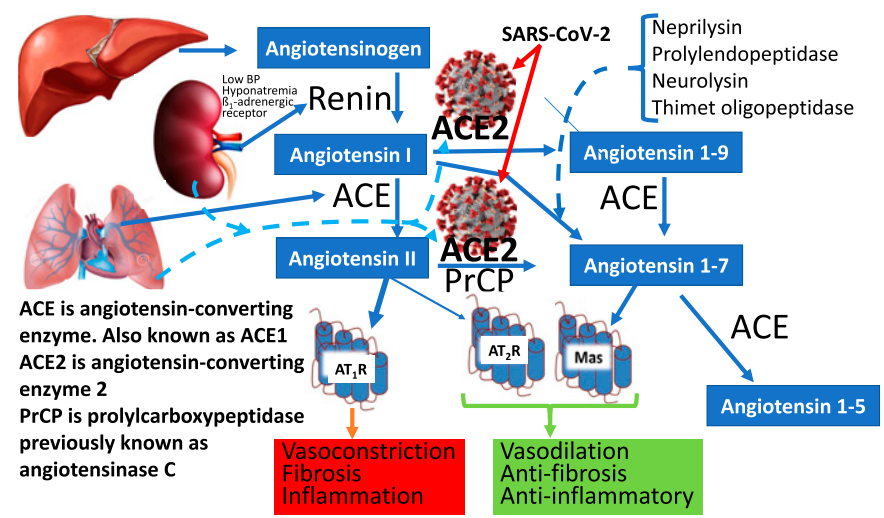

